\title{
On the force drop off phenomenon in shaker testing in experimental modal analysis
}

\author{
Paulo Sergio Varoto* and Leopoldo Pisanelli Rodrigues de Oliveira \\ Dynamics Laboratory, Mechanical Engineering Department, School of Engineering of Sao Carlos, University of \\ Sao Paulo, Brazil
}

\begin{abstract}
The Electrodynamic Vibration Exciter (shakers) has been one of the most employed excitation sources in modal tests. The shaker is an electromechanical device that provides a mechanical motion due to the input signal sent to its coil. Despite being widely used, it is well known that the shaker interacts with the structure under test. In particular, when the structure passes through a given resonance, the force delivered by the shaker abruptly decreases, causing the so called drop off phenomenon. This paper aims to study this force drop off phenomenon in the single shaker modal testing. Analytical models are developed to help in understanding the physical principles involved in the interaction between the shaker and the structure under test. Experimental analyses are performed using different shakers as well as excitation signals, in order to evaluate the effects of the input signal, as well as the power amplifier operational modes, on the structure dynamics. Preliminary tests revealed that significant distortions might occur during vibration tests using shakers and these distortions significantly affect the determination of the structure response.
\end{abstract}

Keywords: Modal testing, mechanical vibration, shaker, force drop off

\section{Introduction}

The electrodynamic vibration exciter, popularly called shaker, is one of the most used power source devices in vibration tests, and one reason for that is the variety of input signals it can provide. It is a very common practice to attach the exciter to the Structure Under Test (SUT) through a flexible stinger which is used to transmit the excitation signals to the SUT in a single direction, reducing secondary forms of excitation (e.g, bending moments) due to possible misalignments [1]. The force transducer is used along with the exciter to measure the input force applied to the SUT. It is usually mounted between the stinger and the SUT so it can measure the input force. In some vibration testing, the

* Corresponding author: Paulo Sergio Varoto, Dynamics Laboratory, Mechanical Engineering Department, School of Engineering of Sao Carlos, University of Sao Paulo, Av. do Trabalhador São-Carlense, 400 São Carlos - S.P. CEP:13566-590, Brazil. Tel.: +55162739423 / 273 9399; Fax: +55 16273 9402; E-mail: varoto@sc.usp.br.
SUT is attached to the exciter table through a test fixture [2]. In this case, the SUT is driven by base excitation signals that are transmitted to the SUT through the test fixture. In this type of test it is common practice to employ a closed loop test procedure where the exciter's table is controlled so that a signal having a prescribed frequency content is applied to the SUT. In all of these cases, it is well known that the exciter interacts with the SUT, and that in some circumstances, distortions specially due to the armature's electrodynamics can alter significantly the measured data.

The exciter dynamics and its interactions with the SUT has been approached by several authors. Tomlinson [6] studied the interaction between the exciter and the SUT, paying special attention to the force drop out that occurs when the structure is excited in the vicinity of a structural natural frequency. This work also emphasizes the non-linearity characteristics that arise from the electromagnetic field that is generated during the exciter working cycle. Olsen [4] studied the effects of the armature's mass and suspension stiffness on the measured data, and pointed out that the armature's mass 
effects on the measured Frequency Response Functions (FRF) can be minimized by selecting an appropriate exciter for a given test. Rao [5] followed in his work the basic development by Olsen, but the drop out of the excitation force has been analyzed in more details. According to his work the drop out phenomenon is primarily due to two causes, namely, the mechanical interaction between the armature's mass and the structure, and the electromagnetic characteristics of the exciter coil circuit.

The exciter dynamics as well as its interactions with the SUT has been approached in different number of text books in modal and vibration testing. Ewins [1] formulated a simple dynamic model that explains the basic mechanical interaction between the exciter's armature and the SUT. The author draws the attention to the fact that the excitation force must be directly measured during the test, in order to obtain reliable FRF measurements. McConnell [2] developed extensive study on the exciter dynamics and its interaction with the SUT. In his work several analytical models were developed to explain the mechanical interactions between the exciter and free as well as grounded SUT. In addition, his work approached the electric characteristics of the exciter-power amplifier system during the test, showing the basic differences that occur when the power amplifier is used either in its voltage or current modes of operation. Maia [?] also developed an interesting study on the subject by using simple dynamic models to model the interactions between the exciter and grounded structures. An insightful paper was published by Lang [3] where several simple tests are performed in order to evaluate the exciter's dynamics.

The objective of the present work is to perform an experimental study on two different vibration exciters, attempting to evaluate some of their basic dynamic characteristics as well as their interaction with the test environment when the force drop out phenomenon is concerned.

\section{Review of theory}

The next section presents a review of important theoretical aspects that are concerned with the dynamics of the vibration exciter. The theoretical development that is described in this section is based on the work by McConnell [2] that presents a comprehensive analytical development on the exciter dynamics. The theoretical development described here considers the exciter's armature dynamics as well as the electrodynamic rela- tionships that are needed to explain the exciter's electric behaviour and the two modes of operation of the power amplifier, the voltage and current modes.

\section{Armature dynamics}

Figure 1 shows the armature dynamic model that consists of the table and the electromagnetic coil. In this case, the exciter base is considered to be rigidly attached to the floor. Hence, we have the 2 DOF mechanical system shown in Fig. 1 , where $m_{1}, k_{1}$ and $c_{1}$ represent the table mass, stiffness and viscous damping and $m_{2}, k_{2}$, and $c_{2}$ the spider mass, stiffness and viscous damping, respectively.

As described in McConnell [2], the frequency domain accelerations $A_{1}(\omega)$ and $A_{2}(\omega)$ exhibited by the table and the coil respectively, can be written in terms of the driving point and transfer accelerance FRFs, $A_{11}, A_{12}$ and $A_{22}$, where, according to Eq. (1), $A_{11}(\omega)$ is the table driving point FRF and $A_{12}(\omega)=A_{21}(\omega)$ is the transfer accelerance FRF.

$$
\begin{aligned}
& A_{1}=\frac{A_{11}(\omega)}{m_{1}+m_{2}} F_{1}+\frac{A_{12}(\omega)}{m_{1}+m_{2}} F_{2} \text { and } \\
& A_{2}=\frac{A_{21}(\omega)}{m_{1}+m_{2}} F_{1}+\frac{A_{22}(\omega)}{m_{1}+m_{2}} F_{2} \text { and }
\end{aligned}
$$

and

The armature's FRFs present in Eq. (1) represent important quantities since they give some useful information about the armature behaviour. The driving point and transfer accelerances $A_{11}(\omega)$ and $A_{22}(\omega)$ are expressed as [2]

$$
\begin{aligned}
A_{11}(\omega) & =\frac{\left(m_{1}+m_{2}\right) A_{1}}{F_{1}} \\
& =\frac{-r^{2}(1+M)\left(\beta^{2}-r^{2}+i \beta \eta_{2}\right)}{\Delta(r)} \\
A_{22}(\omega) & =\frac{\left(m_{1}+m_{2}\right) A_{2}}{F_{2}}= \\
-r^{2}(1+M)\left[\left(1+m \beta^{2}\right)-r^{2}+i\left(\eta_{1}+\beta M \eta_{2}\right)\right] & \Delta(r) \\
A_{12}(\omega) & =\frac{\left(m_{1}+m_{2}\right) A_{1}}{F_{2}} \\
& =\frac{-r^{2}(1+M)\left(\beta^{2}+i \beta \eta_{2}\right)}{\Delta(r)}
\end{aligned}
$$

where the auxiliary variables appearing on Eqs (2), (3) and (4) are defined as 


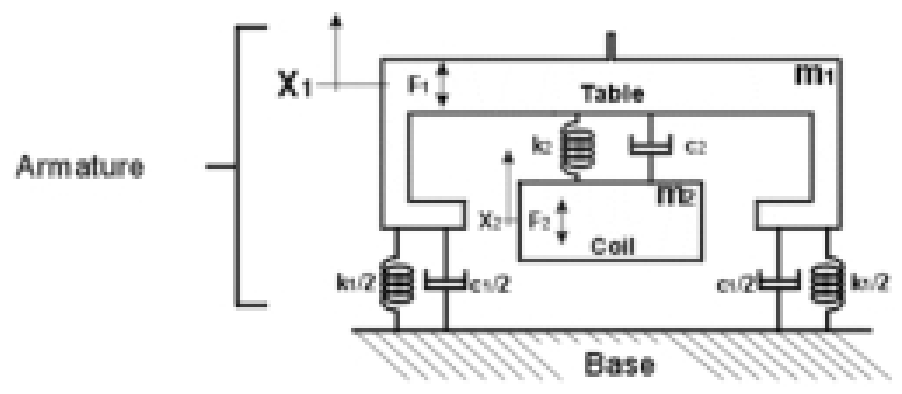

Fig. 1. Armature 2 DOF dynamic model.

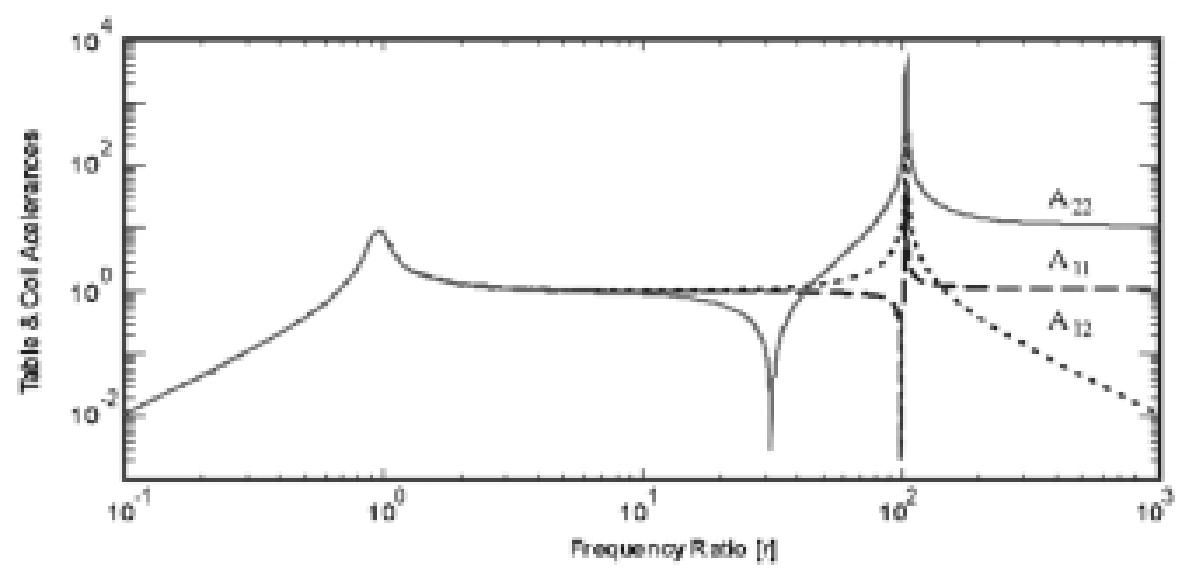

Fig. 2. Dimensionless accelerance plot for exciter armature.

$$
\begin{aligned}
\beta=\frac{\omega_{22}}{\omega_{11}} & M=\frac{m_{2}}{m_{1}} \\
\Delta(r)= & {\left[\left(1+\beta^{2} M\right)-r^{2}+j 2 r\left(\zeta_{1}+\zeta_{2} \beta M\right)\right] } \\
& {\left[\beta^{2}-r^{2}+j 2 r \zeta_{2} \beta\right] } \\
& -\left[\beta^{2} M\right]\left[\beta+j 2 r \zeta_{2}\right]^{2}
\end{aligned}
$$

These tree dimensionless accelerances FRFs are shown in Fig. 2. They are identical as the dimensionless frequency ratio $r$ ranges from 0.1 to 10 . In this frequency range it is observed the existence of a natural frequency that is common to all FRF and that is the first resonance of the two DOF system. Above $r=$ 10.0, the FRFs diverge and each one exhibits their own characteristics.

The coils accelerance $A_{22}(\omega)$, decreases after $r=$ 10 up to $r \cong 31.6$, where the table presents a dynamic absorber type of behaviour for the coil. Then, this accelerance increases up to $r \cong 105$, (coil's resonance), and become s constant ( 11 or $20.8 \mathrm{~dB}$ ).

The table driving point accelerance $A_{11}(\omega)$ exhibits an anti-resonance at $r=\beta=100$ followed by its resonance at $r \cong 105$ and reaches a plateau of 1.1 (i.e. $1+M)$ for higher frequencies $(r \gg 100)$.

Finally, considering table transfer accelerance $A_{12}$ $(\omega)$, we have a resonance at $r \cong 105$ and then it decreases at a ratio of $40 \mathrm{~dB} /$ decade. This behaviour showed by $A_{12}(\omega)$ clearly indicates that it is quite impossible to control the table after $r=300$, since its dynamic response is more affected by external forces than by the coils input. Therefore, the armature-coil system has an upper frequency limit for effective use of the exciter. In this case this frequency limit is given by [2]:

$$
\begin{aligned}
r_{2} & =\beta \sqrt{1+M}=\frac{\omega_{22}}{\omega_{11}} \sqrt{1+M} \\
& =\sqrt{\frac{k_{2}\left(m_{1}+m_{2}\right)}{k_{1} m_{2}}}
\end{aligned}
$$

Figure 3 shows the electromechanical model used by Olsem [4] and McConnell [2] to describe the electromagnetic coupling on the armature-coil system. This electromechanical coupling is governed by several parameter, as for example the coil resistance $R$ and in- 

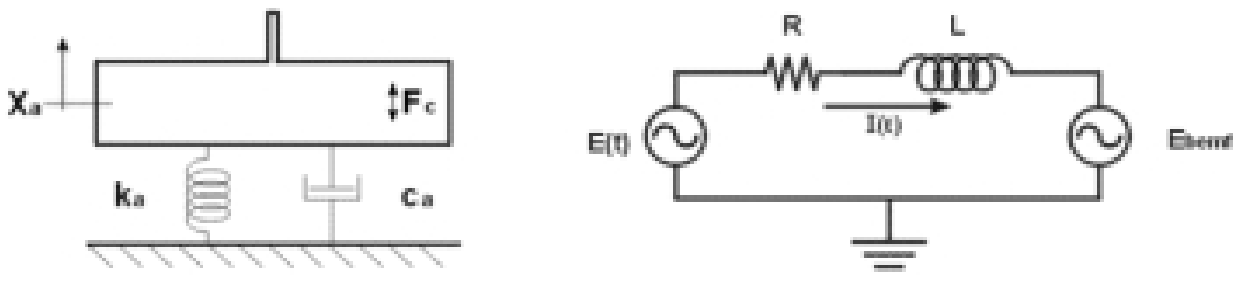

Fig. 3. Exciter's electromechanical model.

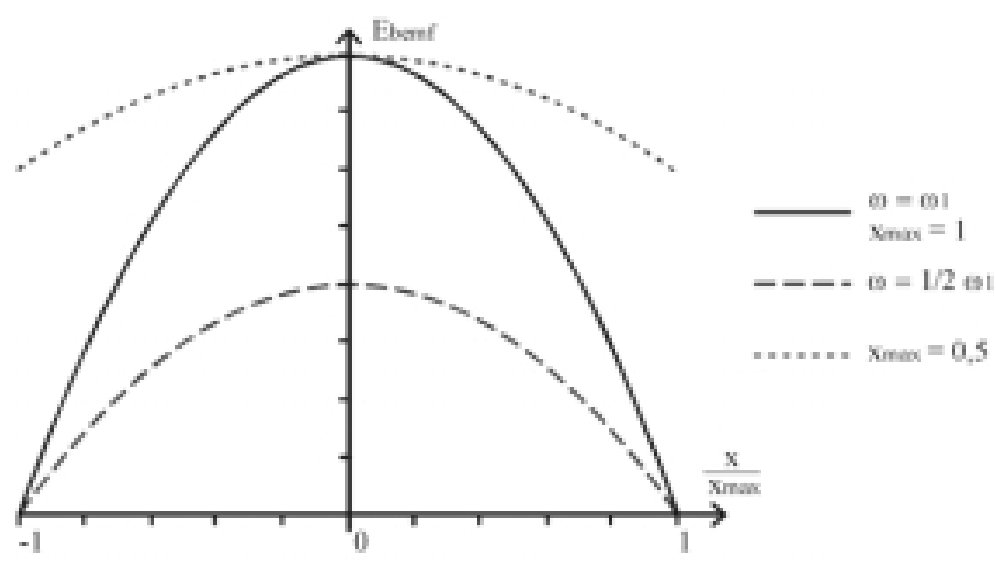

Fig. 4. $E_{\text {bemf }}$ behaviour due to frequency and amplitude variation.

ductance $L$, and the input voltage signal $E(t)$ and the back electromagnetic voltage $E_{\text {bemf }}$.

The equations that govern the mechanical and electrical systems shown in Fig. 3 are respectively given as

$$
\begin{aligned}
& m_{a} \ddot{x}+c_{a} \ddot{x}+k_{a} x=F_{c}(t) \\
& R O+L \dot{I}+E_{\mathrm{bemf}}=E(t)
\end{aligned}
$$

where Eq. (9) was obtained using standard electric circuits relationships. However, the electromagnetic force $F_{c}(t)$, as well as the Ebemf voltage are hardly dependent on the exciter's mode of operation [2]. The subsequent sections shows the basic equations for both cases, voltage and current mode.

The electrodynamic phenomenon that occurs on the exciter's circuits is mainly due to the interaction between the current and the armature motion in the exciter's electromagnetic field.

The Ampère law relates the electromagnetic force $F_{c}(t)$ and the current $I(t)$ through the coil, according to

$$
F_{c}=(n B l) I=K_{f} I
$$

where $B$ is the magnetic field intensity generated by the current $\mathrm{I}(\mathrm{t})$ through the $n$ coils, each one having length equal to $l$. The constant $K_{f}$, is the 'force-current constant' and is equal to $(n B l)$.

Tomlinson [6] developed a theoretical model establishing a relationship between the magnetic field $B$ and the coil position $x$ given as

$$
B=\frac{d \psi}{d x}=B_{0}\left[1-\left(\frac{x+x_{0}}{x_{\max }}\right)^{2}\right]
$$

where $B_{0}$ is the highest intensity that the field $B$ reaches, $x_{0}$ is the armature initial position, $x$ is its oscillation amplitude and $x_{\max }$ its maximum amplitude. It can be verified yet, by Eqs (10) and (11) that the relationship between the force and the current is non linear

$$
F_{c}=n l B_{0}\left[1-\left(\frac{x+x_{0}}{x_{\max }}\right)^{2}\right] I
$$

In addition, Lenz law gives the relationship between the $E_{\text {bemf }}$ and the armature's velocity as:

$$
\begin{aligned}
E_{\mathrm{bemf}} & =n l B \dot{x}=n l B_{o}\left[1-\left(\frac{x+x_{0}}{x_{\max }}\right)^{2}\right] \dot{x} \\
& =K_{v}\left[1-\left(\frac{x+x_{0}}{x_{\max }}\right)^{2}\right] \dot{x}
\end{aligned}
$$


It could be noticed from Eq. (13) that the $E_{\text {bemf }}$ also presents a nonlinear factor proportional to the relation between the excitation amplitude and the armature maximum amplitude, and its velocity, i.e., the excitation frequency [6].

Figure 4 shows the $E_{\text {bemf }}$ behaviour in terms of the excitation amplitude and frequency. When the frequency is half of the original excitation frequency, the $E_{\text {bemf }}$ drops to a new value that is half of the original one. The effects of the amplitude of oscillation are related to the nonlinear behaviour. The smaller the amplitude, more linear is the $E_{\text {bemf }}$ variation [2].

\subsection{Power amplifier-modes of operation}

As stated early on this paper, the power amplifier has two modes of operation, the current and the voltage modes, respectively. These modes establish the voltage vs. current relationships during the exciter operation. The basics of each mode of operation will be described as follows.

In the current mode of operation, the relationship between the input voltage $(V)$ to the amplifier and its output current $(I)$ is given by an equation of the type:

$$
I(\omega)=G_{i}(\omega) V(\omega)
$$

where $G_{i}$ is the amplifier current mode gain.

The frequency domain versions of Eqs (8) and (9) are given as

$$
\begin{aligned}
& \left(k_{a}-m_{a} \omega^{2}+j c_{a} \omega\right) X=K_{f} I_{0} \\
& (R+j L \omega) I_{0}+j K_{v} \omega X=E_{0}
\end{aligned}
$$

where $I_{o}$ and $E_{0}$ are reference amplitudes for the current and voltage, respectively. Notice that the simpler relationship between the $E_{\text {bemf }}$ and the table's velocity (Eq. (13)) is used in Eq. (16).

The dimensionless armature accelerance in the current mode is given by:

$$
A(\omega)=\frac{m_{a}\left(-\omega^{2} X\right)}{K_{f} I_{0}}=\frac{-r^{2}}{1-r^{2}+j 2 \zeta_{a} r}
$$

where $r$ is the dimensionless frequency ratio, now based on the armature natural frequency, $\zeta_{a}$ is the armature viscous damping ratio.

Thus, the voltage needed to maintain the current magnitude is given as

$$
\begin{aligned}
E(\omega) & =\frac{E_{0}}{R I_{0}} \\
& =1+j\left[\beta_{1}+\frac{2 \zeta_{e}}{1-r^{2}+j 2 \zeta_{a} r}\right]
\end{aligned}
$$

Table 1

MB dynamics shaker features

\begin{tabular}{ll}
\hline Feature & Value \\
\hline Force $(\mathrm{N})$ & 111 \\
Useful Displacement $(\mathrm{mm})$ & 25.4 \\
Maximum Displacement $(\mathrm{mm})$ & 27.9 \\
Shaker mass $(\mathrm{kg})$ & 24.9 \\
Armature mass $(\mathrm{kg})$ & 0.227 \\
Armature axial stiffness $(\mathrm{N} / \mathrm{m})$ & 2312 \\
Max. Coil Current $(\mathrm{A})$ & 8.5 (low impedance) \\
& 4.2 (high Impedance) \\
Coil resistance $(\Omega)$ & 1.3 (low impedance) \\
& 5.2 (high Impedance) \\
\hline
\end{tabular}

where $\beta_{1}=\omega_{a} / \omega_{e}$ and $\omega_{a}$ is the armature's natural frequency and $\omega_{e}$ is the break frequency:

$$
\omega_{e}=\frac{R}{L}
$$

The electromagnetic damping ratio $\zeta_{e}$ is given by:

$$
\zeta_{e}=\frac{C_{m}}{2 \sqrt{k_{a} m_{a}}}=\frac{K_{v} K_{f}}{2 R \sqrt{k_{a} m_{a}}}
$$

which is the result of the back emf current being dissipated by the coil circuit.

It is important to remember that this equations are valid for low table amplitudes, which makes the nonlinear terms vanish, as described by Tomlinson.

Similarly to the current mode, in the voltage mode of operation, the power amplifier can be modelled as a gain, constant in frequency up to a given cutoff frequency [2], according to the following equation

$$
E(\omega)=G_{v}(\omega) V(\omega)
$$

where $G_{v}(w)$ is the amplifier gain, $V(\omega)$ and $E(\omega)$ are the amplifier's input and output voltages, respectively.

Following a similar procedure, the dimensionless armature accelerance in the voltage mode of operation is give as:

$$
\begin{aligned}
& A(\omega)=\frac{m_{a}\left(\omega^{2} X\right)}{K_{f}\left(E_{0} / R\right)}= \\
& \frac{-r^{2}}{1-\left(1+M_{L}\right) r^{2}+j\left[2\left(\zeta_{a}+\zeta_{e}\right)+\beta\left(1-r^{2}\right)\right] r}
\end{aligned}
$$

where the dimensionless mass ratio $M_{L}=m_{e} / m_{a}$ and the inductive mass $m_{e}$ is given by:

$$
m_{e}=\frac{L c_{a}}{R}
$$

where it can be noticed from this last equation that the inductive mass is dependent of the armature damping.

In order to gain additional insights on the exciter's dynamic behaviour when operated in either the current or voltage modes, a simple simulation was performed 


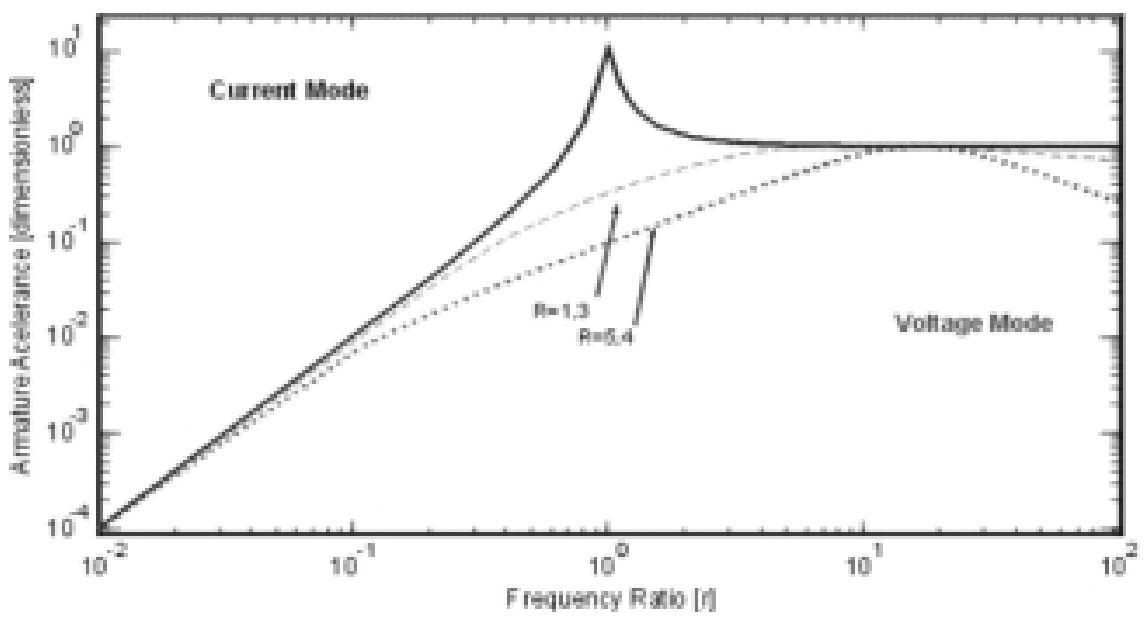

Fig. 5. Dimensionless bare table accelerance.
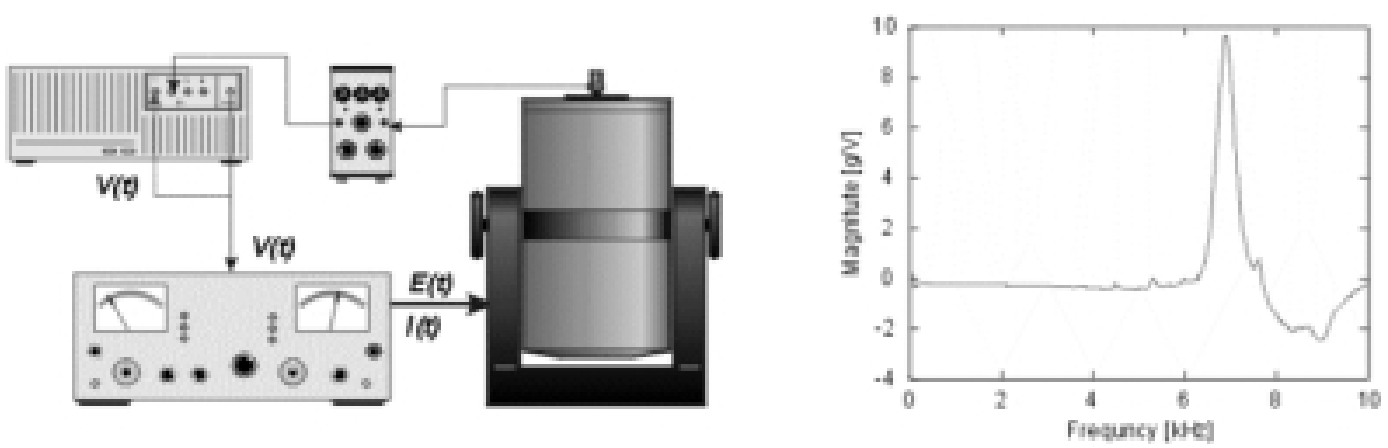

Fig. 6. B\&K 4812 bare table FRF.

with the physical parameters of an available vibration exciter. The exciter parameters are shown below and correspond to a MB Dynamics Modal 50 vibration exciter. Table 1 shows these parameters were obtained from the exciter's operating manual.

Figure 5 shows the bare armature accelerance FRF behaviour for the current and voltage modes, according to Eqs (17) and (22), where it is clearly seen the differences between the two modes of operation. In the current mode FRF (solid line), once the table passes through its mechanical resonance, the FRF amplitude becomes constant, and this implies in principle a reliable condition for the exciter operation, as stated by McConnell [2]. The other two FRFs depicted on Fig. 5 (dashed and dashed-dotted) correspond to the bare table FRF in the voltage mode of operation. There is a clear distinction between these FRFs when compared to the current mode FRF. The bare table resonance is severely damped in the voltage mode FRF, mostly due to the high electrodynamic damping. Also, the two voltage mode FRFs shown in Fig. 5 are different in the sense that they use different values for the coil resistance. It is seen that the smaller resistance yielded magnitude closer to the current FRF for higher frequencies.

\section{Experimental results}

This section presents some experimental results from tests which were performed in order to get some practical understanding on the exciter's dynamic behaviour as well as on the interaction between the exciter and the SUT. The exciters used during the tests were a B\&K 4812 with Power Amplifier B\&K 2707 and a MB Dynamics Modal 50 with Power Amplifier SL500VCF.

\subsection{Results for Armature's dynamics}

This section shows a sample of experimental results that were obtained in tests that aimed to know the exciter's basic dynamic behaviour. 


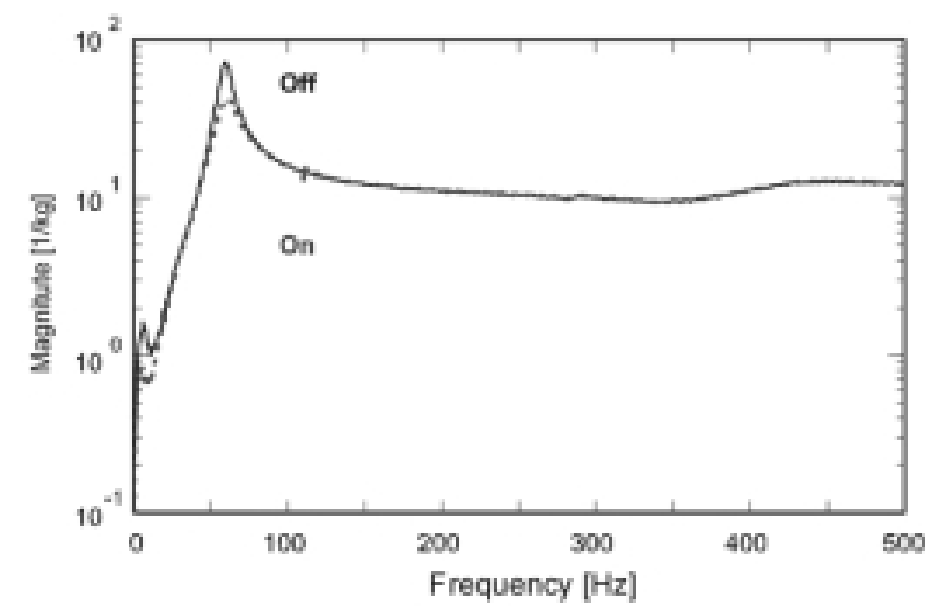

Fig. 7. Electromagnetic damping.

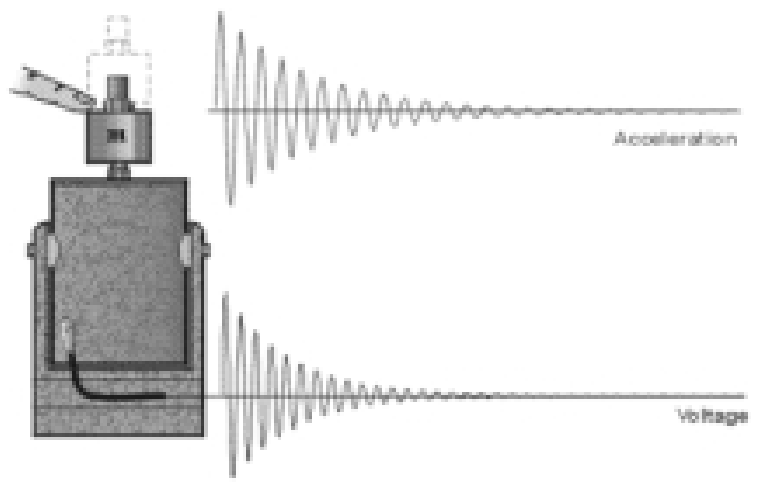

Fig. 8. Armature free response.

Figure 6 depicts the B\&K 4812 vibration exciter plus Power Amplifier B\&K 2707 bare table acceleration FRF. This result was obtained by measuring the exciter's table acceleration using the B\&K 4371 accelerometer while the excitation frequency was varied in the $0-10 \mathrm{kHz}$ frequency range. In this test the shaker was fed by a random signal, covering the entire frequency range. Hanning windows were used in all data acquisition channels to reduce filter leakage. The acceleration values were normalized by the input voltage from the signal generator. The result shown in Fig. 6 exhibits peak frequency at $6,885 \mathrm{~Hz}$, while the exciter operating manual gives $7,200 \mathrm{~Hz}$ as the bare table natural frequency. Hence, although there is a difference of about $4 \%$, this test was considered effective in obtaining the bare table natural frequency.

Figure 7 shows results obtained when the shaker table is impacted by an instrumented hammer and the table acceleration is measured in two different conditions. The solid line shows the FRF that was obtained when the power amplifier is turned off, and the dashed line shows the same FRF, that this time was obtained with the power amplifier turned on. In this condition no excitation signals were sent to the shaker, the only difference between these test conditions is that in the first case the exciter's internal circuits are not electrically energized while in the second the power amplifier is turned on and thus electrical energy is flowing through the coil circuit. The results shown in Fig. 7 are suitable for observing the effects of the additional damping and possibly stiffness induced by the coil electromagnetic field that is established during the exciter operation.

Figure 8 shows a simple but interesting test that was performed with the MB Dynamics Modal 50 exciter in order to get an estimate of the armature's suspension damping ratio. A small mass with a miniature accelerometer was mounted in the top of the exciter table and with the power amplifier off, the armature was plucked and the free decay acceleration was measured. This data was used estimate the damping factor through the logarithmic decrement, resulting a value of $2.6 \%$ for the armature's damping ratio.

\subsection{Results for exciter-SUT interaction}

This section shows a sample among many results obtained in the developed project. The results here shown contain important features concerned with the exciter-SUT interaction.

Figure 9 shows the experimental setup used in one of the tests. This test used a cold-rolled steel beam (1000 $\times 25.4 \times 6.25 \mathrm{~mm}$ ) mounted on the MB Dynamics exciter table through a stinger. A Kistler 912 force transducer was used to measure the input force to the 


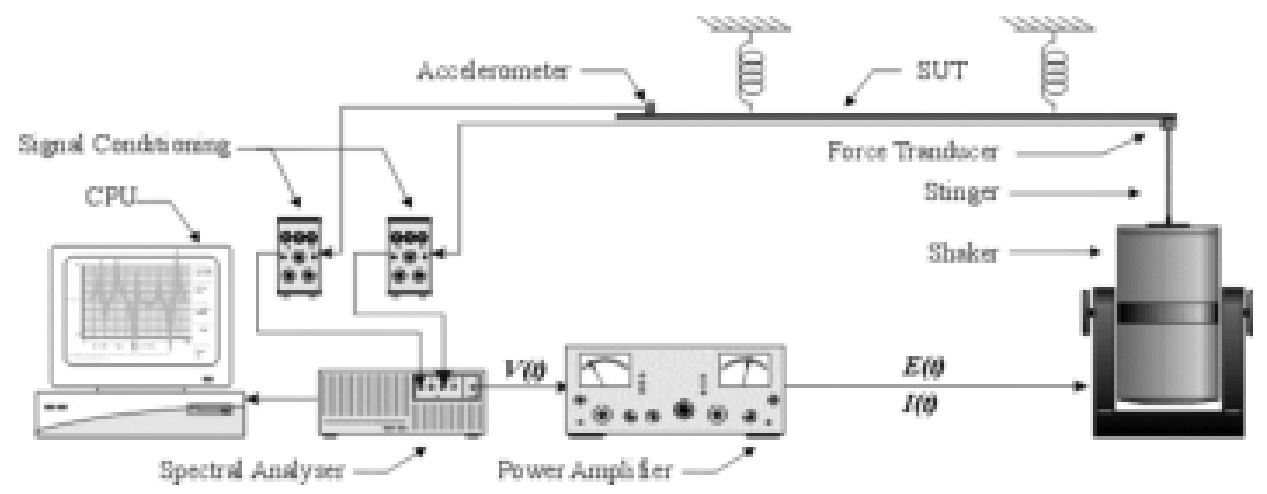

Fig. 9. Test on free - free steel beam.

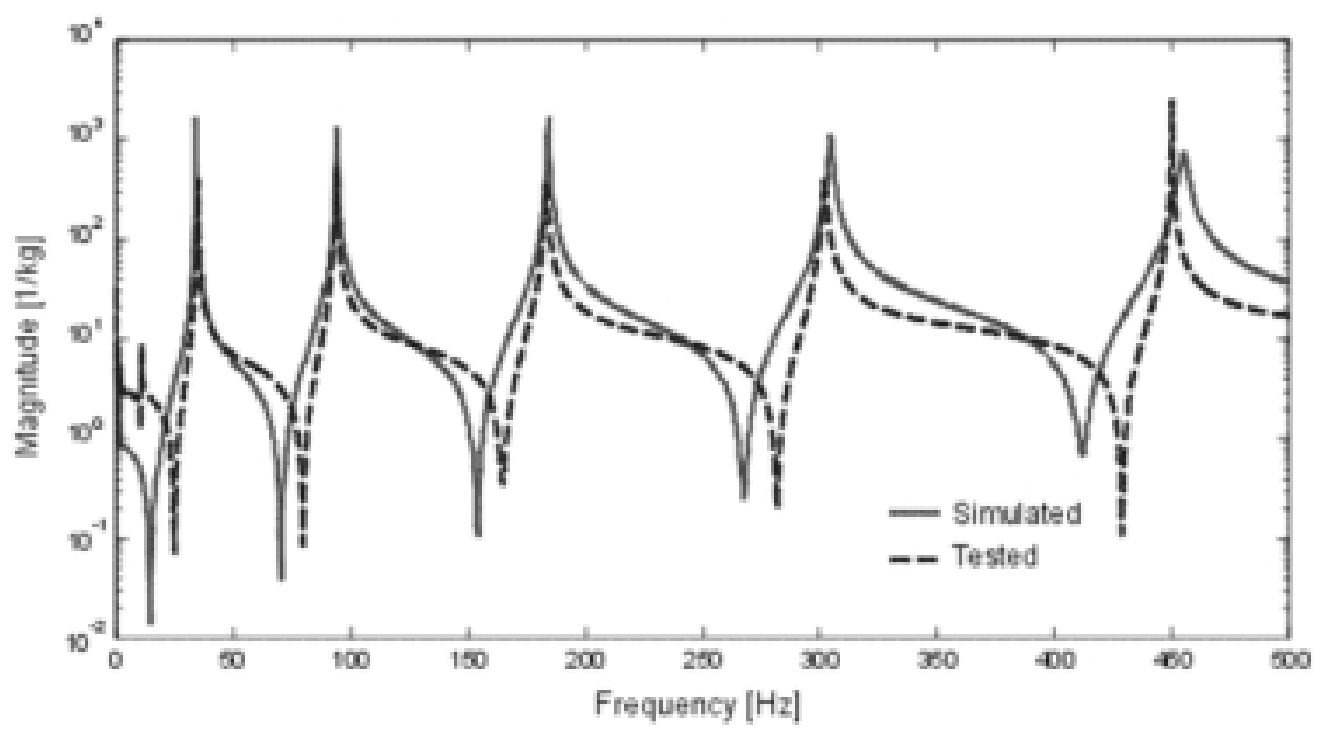

Fig. 10. Driving point FRFs.

beam, and a B\&K 4375 accelerometer was used to measure the beam's output acceleration. The beam was excited with a random as well as a chirp signal in the $0-500 \mathrm{~Hz}$ frequency range. The SL500VCF power amplifier was adjusted to operate in the voltage and current modes.

A numerical model of the beam/exciter system depicted in Fig. 9 has been developed so as it can be possible to study the exciter SUT interaction phenomenon features. The beam was modeled as a continuous system by using the standard fourth order partial differential equation. First, the beam's mode shape eigen functions were obtained from the solution of the partial differential equation with free-free boundary conditions by using the separation of variables approach. Second, the beam's driving point and transfer FRF were obtained by the superposition method. Then, the in- teraction with the shaker was calculated by assuming a SDOF lumped dynamic model for the B\&K 4812 exciter. The exciter's model consants were obtained from previous experiments. This model was then connected to the beam's model in the frequency domain by using acceleration transmissibilty conceprs. Figure 10 shows the driven point FRFs obtained from this numerical simulation and the performed test. Despite the fact that the anti-resonance did not match exactly, due to a number of reasons, the resonant peaks showed a good correlation when frequency and magnitude are concerned.

Figure 11 shows the results obtained for the input force to the beam for the voltage mode of operation. The numerical simulation is compared to the input force measured during a random and a chirp excitation. It is noticed a dropout on force values at frequencies in the 


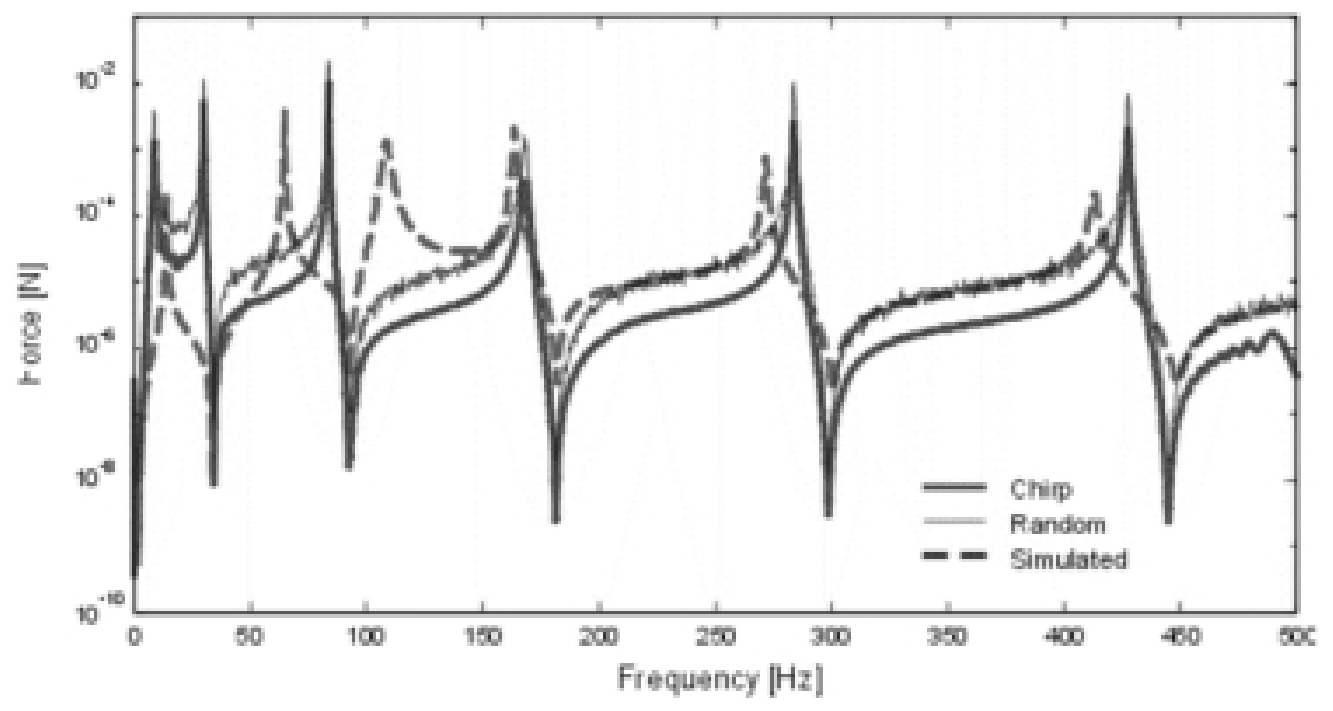

Fig. 11. Input force - voltage mode.

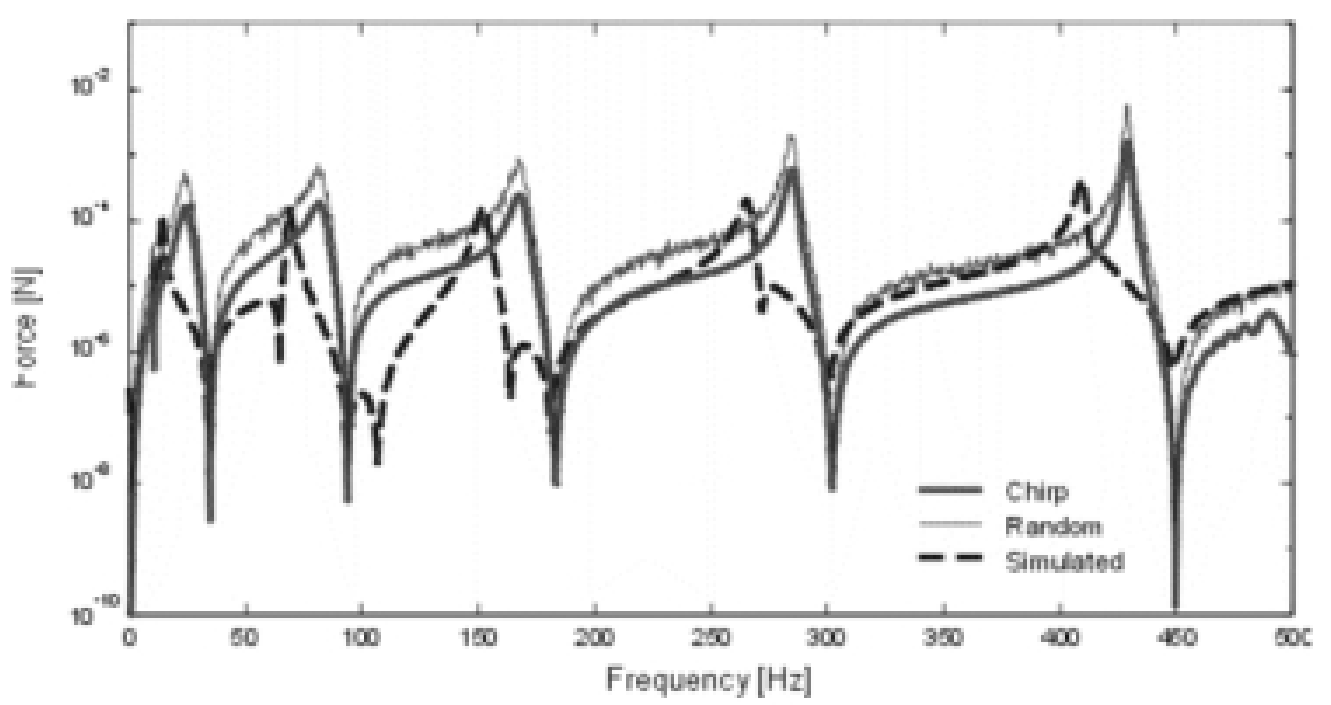

Fig. 12. Input force - current mode.

vicinity of the beam's ungrounded natural frequencies. Similar behaviour was observed by McConnell [2] in numerically simulated results.

Figure 12 shows the same results obtained for the current mode of operation. Special attention should be paid during current mode tests mainly when the SUT is light and flexible. High levels of force and voltage can be reached due to the low electrodynamic damping related to this type of operation. The same observations were made by other authors $[1,2,5]$.

Figure 13(a) shows an accelerance FRF that was obtained using the B\&K 4812 exciter with random exci- tation and the PCB impact hammer. In this case, a simpler structure was used, that contains a single natural frequency in the $0-100 \mathrm{~Hz}$ frequency range. The experimental results are compared with results obtained from an analytical model of SUT.

The main feature of this test is that even though two different excitation mechanisms were employed to drive the test structure, the same value is obtained for the natural frequency (about $27.3 \mathrm{~Hz}$ ), as shown in Fig. 13(a). Recall that, although not shown here, in impact testing the input force auto spectral density is constant (or at least nearly constant!) in the tested 

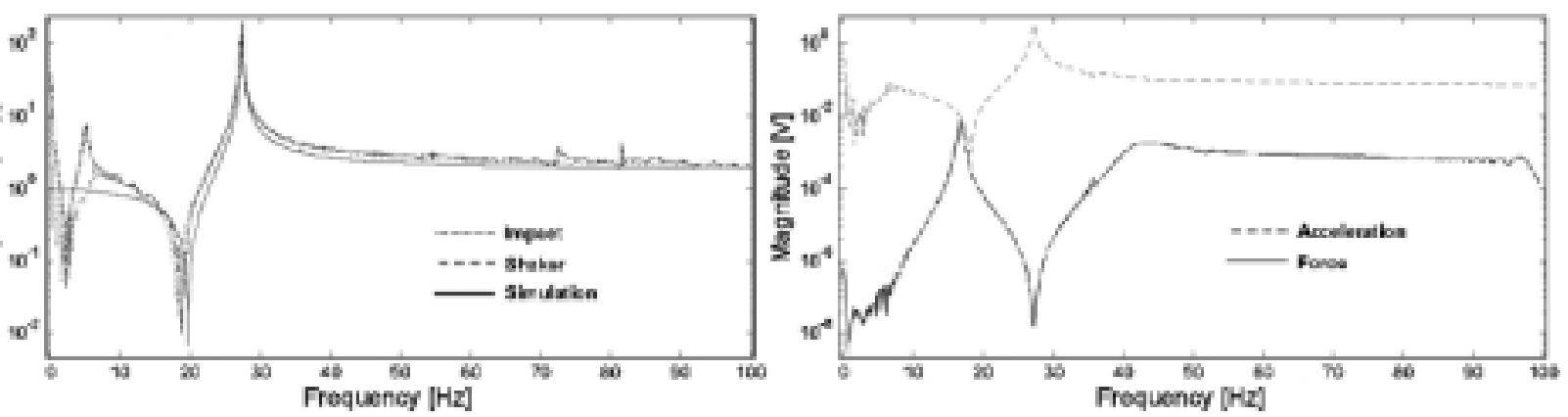

Fig. 13. (a) Driving point FRFs for a free-free 2DOF system (b) Driving point acceleration and the input force.

frequency range, not presenting the glitches that appear in the shaker input signal. Figure 13(a) still shows a rigid body natural frequency (about $5 \mathrm{~Hz}$ ) due to the SUT suspension system that is not present in the ideal simulated FRF.

It can still be noticed that although the natural frequencies are essentially the same for both testing conditions, there is mismatch in the anti-resonance, which occurs at a frequency close to $20 \mathrm{~Hz}$. An interesting fact can be observed in the anti-resonance obtained in the exciter test. The anti-resonance (dashed line) occurs at approximately $18 \mathrm{~Hz}$, a value that is slightly lower than the anti-resonance value for the hammer test (dotted line). The armature's suspension stiffness and mass values for the $\mathrm{B} \& \mathrm{~K}$ exciter are about $K_{a}=21000 \mathrm{~N} / \mathrm{m}$ and $m_{a}=0.454 \mathrm{Kg}$, as stated in the operating manual. On the other hand, the SUT has a mass of $m=1.046 \mathrm{Kg}$. These values allow us to get the frequency:

$$
\begin{aligned}
\omega & =\sqrt{k_{a} /\left(m+m_{a}\right)}=118.3 \mathrm{rd} / \mathrm{s} \\
& =18.83 \mathrm{~Hz}
\end{aligned}
$$

Hence, the anti-resonance observed in Fig. 13(a) is essentially the natural frequency of the armature-SUT system, but in the SUT response it appears as dynamic absorber type of action. Finally, the anti-resonance amplitude obtained with the exciter testing is higher than that obtained with impact testing, a result that, along with the frequency mismatch already discussed indicates a clear exciter-SUT interaction. Figure 13(b) shows simultaneously a plot of the structure acceleration and the input force. These quantities were normalized so that they could be plotted in the same graph. It shows essentially the same behaviour observed in Fig. 11. Once again, it can be notice that the value of the structure's natural frequency and the frequency where the force drops out are very close.

\section{Summary and conclusions}

This paper performed an analytical and experimental study on the exciter dynamic behaviour as well as its interaction with the structure under test. The tests performed allowed observation of interesting characteristics when the shaker dynamic and its interaction with the SUT are concern. The major conclusion from this work is that the exciter represents an effective excitation mechanism, which however, should be used with care, since it interacts with the test environment. Special attention should be paid to the power amplifier, since its two modes of operation can cause distortions on the test results, specially due to the electromagnetic damping, that is difficult to estimate due to the non linear behaviour of the magnetic field on the exciter circuits. This occurs mainly during the current mode of operation that has shown bigger amplitudes of vibration due to the very low electrodynamic damping, which either can result in oversets. The results shown on this paper represents a sample of a more detailed investigation that has been continuously developed by the authors, and new results, related to multi shaker tests, will be available in a near future.

\section{Acknowledgements}

The authors acknowledge the financial support provided by FAPESP (grants \# 99/0229-6 and 00/14700-4) to Mr. Leopoldo P. R. de Oliveira during the development of the project, and to EESC-USP for providing the necessary laboratory facilities.

\section{References}

[1] D.J. Ewins, Modal Testing: Theory and Practice, RPS, London, UK, 1984. 
[2] K.G. McConnell, Vibration Testing: Theory and Practice, John Wiley \& Sons, NY, EUA, 1995.

[3] G. Lang, Electrodynamics Shakers Fundamentals, Sound and Vibration Magazine (April 1997), 14-25.

[4] N.L. Olsem, Using and Understanding Electrodynamic Shakers in Modal Applications, Proceedings of the 4th International Modal Analysis Conference, IMAC 2 (1986), 1160-1167.

[5] D.K. Rao, Electrodynamic Interaction Between a Resonating Structure and an Exciter, Proceedings of the 5th International Modal Analysis Conference, IMAC 2 (1987), 1142-1150.
[6] G.R. Tomlinson, Force Distortion in Resonance Testing of Structures with Electrodynamic Vibration Exciters, Journal of Sound and Vibration 63(30) (1979), 337-350.

[7] P.S. Varoto and L.P.R. Oliveira, On the Interaction Between the Vibration Exciter and the Structure Under Test in Vibration Testing, Proceedings of the 19tn International Modal Analysis Conference, IMAC 2 (2000), 1142-1150.

[8] Instruction Manual, B\&K Vibration Exciter System V, 1974.

[9] Instruction Manual, MB Dynamics Modal 50A vibration Exciter, 2000. 

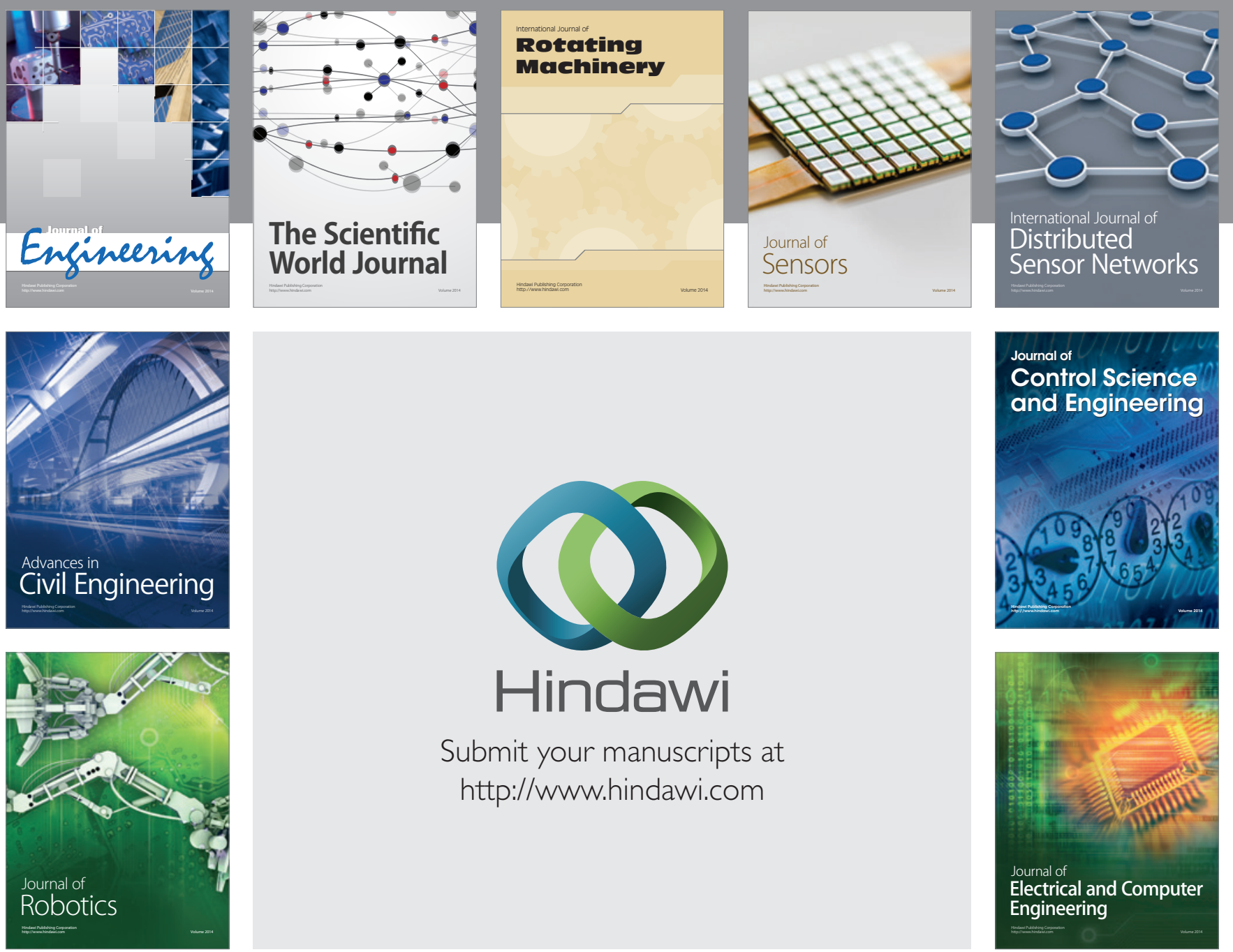

Submit your manuscripts at

http://www.hindawi.com
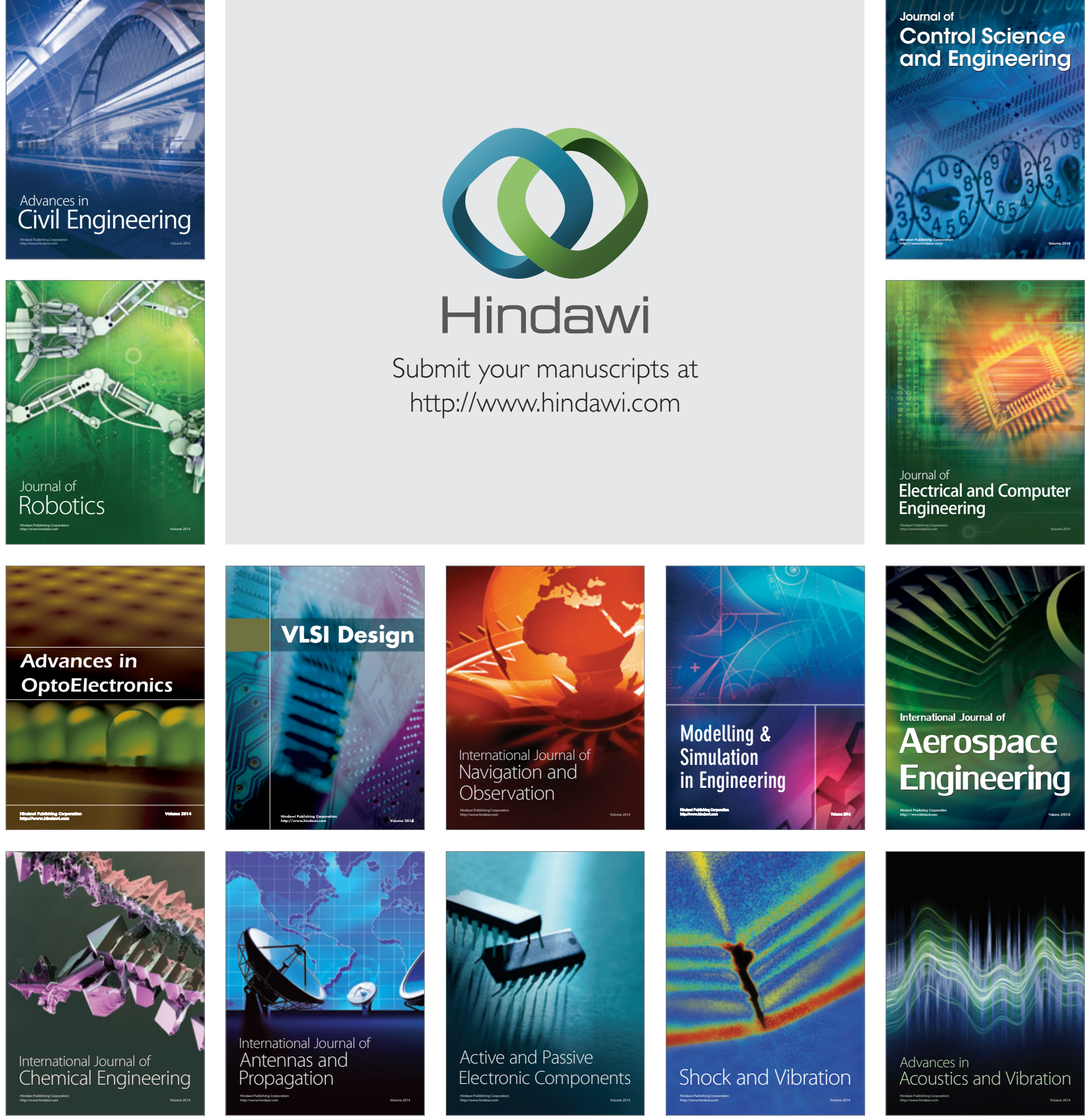